\title{
Technology-Enhanced Faculty Development: Future Trends and Possibilities for Health Sciences Education
}

\author{
Yusuf Yilmaz ${ }^{1,2}$ (D) Sarrah Lal ${ }^{1,3,4} \cdot$ X. Catherine Tong ${ }^{5} \cdot$ Michelle Howard $^{5} \cdot$ Sharon Bal $^{5} \cdot$ Ilana Bayer $^{3,6}$. \\ Sandra Monteiro ${ }^{1,7} \cdot$ Teresa M. Chan ${ }^{1,8,9}$ (I)
}

Accepted: 1 October 2020 / Published online: 14 October 2020

(C) International Association of Medical Science Educators 2020

\section{Introduction}

Engagement is the focus of technology-enhanced faculty development. Unfortunately, faculty development (i.e. "teaching the teacher") can sometimes fail to mirror the advances we have created for our students, leaving room for innovation, modernization, and change [1]. While research has shown learning outcomes can be somewhat equivocal for traditional approaches to teaching compared to technology-enhanced ones [2-4] sometimes favouring lower technology approaches $[5,6]$, continued use of "the same boring methods" certainly limits the level of learner engagement. Practically speaking, unengaged faculty will not pursue learning objectives. Technology-enhanced faculty development supports various benefits, from access to

Yusuf Yilmaz

yusufyyilmaz@gmail.com

Sarrah Lal

sarrah@mcmaster.ca

X. Catherine Tong

tongxx@mcmaster.ca

Michelle Howard

mhoward@mcmaster.ca

Sharon Bal

bals4@mcmaster.ca

Ilana Bayer

bayer@mcmaster.ca

Sandra Monteiro

monteisd@mcmaster.ca

Teresa M. Chan

teresa.chan@medportal.ca

1 McMaster University Faculty of Health Sciences McMaster Education Research, Innovation and Theory (MERIT) Program, Hamilton, Ontario, Canada content through diverse device options (e.g. mobile phones) to interaction with content via instant feedback options (e.g. interactive video, online quiz). Additionally, with distributed and online education models growing, innovative education technology can vastly improve access to education.

Online learning technologies have been developed for educational contexts (e.g. learning management systems [LMS], elearning authoring tools), but there are also various technologies targeted for public use so that any internet user can access (e.g. search engines, social media, productivity platforms) which may also be incorporated into our faculty lives. Without careful planning, blending multiple technologies can fail to achieve desired faculty learning outcomes - or worse, become a deterrent to faculty using these technologies [1, 7-9]. In this paper,

Department of Medical Education, Faculty of Medicine, Ege University, Izmir, Turkey

3 MacPherson Leadership in Teaching and Learning Fellowship, McMaster University, Hamilton, Ontarion, Canada

4 Michael G. DeGroote Initiative for Innovation in Healthcare and Division of Education \& Innovation, Department of Medicine, McMaster University, Hamilton, Ontario, Canada

5 Department of Family Medicine, McMaster University, Hamilton, Ontario, Canada

6 Department of Pathology and Molecular Medicine, Faculty of Health Sciences Learning Technologies Lab, McMaster University, Hamilton, Ontario, Canada

7 Department of Health Research Methods, Evidence and Impact, McMaster University, Hamilton, Ontario, Canada

8 Program for Faculty Development, Faculty of Health Sciences, McMaster University, Hamilton, Ontario, Canada

9 Division of Emergency Medicine, Department of Medicine, McMaster University, Hamilton, Ontario, Canada 
we discuss both innovation and caution for the use of educational technologies in faculty development. In particular, we propose attending to the educational technology learning needs of faculty as their capacity will influence perceptions [10], acceptance, and adoption of these innovations [11].

Traditional faculty development offerings include seminars, workshops, and longitudinal programs $[12,13]$. These approaches may fail to address the current rapid, on-demand, and self-regulated faculty development needs. As a group of early-to-mid-career educators and faculty development scholars, we have reviewed selected evidence and research around technologies that have not yet substantively impacted the practices of faculty development. Our authorship team comprises a mix of teaching faculty, clinician educators, and education scientists. From our diverse lenses, we aim to highlight trends that might be brought to improve faculty development practices in health sciences education. While we are not proposing that these strategies are the only methods that faculty developers might use for their teaching strategies, we hope they may serve to foster continued auditing of existing modalities to ensure that we continue to evolve faculty development practices in 2020 and beyond.

In this paper, we draw on our expertise to highlight 5 internet-based education strategies that we believe should inform the future of faculty development in health sciences education. Table 1 provides an overview of recommended strategies and how they might impact the future of faculty development for health sciences education. We describe five technology trends within society broadly and re-imagine faculty development with these technologies.

\section{Just-in-Time Learning}

Just-in-time (JiT) refers to engaging in information gathering when the need arises, for example, looking up how to take a screenshot of your smartphone. The strength of Google's search engine has enabled this behaviour; trainees often seek to identify their own knowledge gap and then fill that gap using several sources (e.g. Google [14], YouTube [15], Twitter [16]). Concerns over the veracity of these sources may discourage faculty from engaging with or encouraging reliance on online resources themselves [17]. Additionally, self-assessment of knowledge gaps can be an unreliable driver for learning [18]. However, such searches can be encouraged as just-in-time learning strategies with instructor guidance $[17,19]$ mitigating the limitations of self-assessment, pivoting instead towards using such searches as a method to identify gaps [20].

\section{Leveraging Just-in-Time Faculty Development}

JiT approaches identify teaching and learning activities that identify learners' needs in a timely fashion to provide educational support [21]. There are several naming conventions of JiT such as just-in-time teaching [22, 23], just-in-time learning [24], just-in-time training [25], and just-in-time education [26]. While there are differences in the way each method is applied, we use JiT to refer to a broad strategy in this paper.

Current technology offers JiT solutions to a growing demand for efficient on-the-job learning [27]. Mobile applications can deliver timely and accessible content using different nudging options (e.g. push notifications) [28]. Timelines may be determined through algorithms that analyze clinical practice schedules, teaching responsibilities, and noted knowledge deficiencies (either self-identified or systems-identified) to create customized needs assessments. Therefore, JiT may increase engagement for faculty development by targeting faculty who are receptive to curated material, and ready to learn due to identified knowledge gaps [29].

\section{Evidence of JiT for Health Professionals}

JiT has enabled emergency medicine faculty members to be ready for teaching shifts by presenting best practices for effective feedback [30]. Nelson and colleagues [31] showed that JiT easily adapts to specific needs within various clinical scenarios to train faculty members. Even emails and text messages are as effective as other high-stake technologies to implement JiT in faculty development [32]. Finally, there is some overlap with JiT and harnessing the power of virtual communities of practice $(\mathrm{CoPs})$.

\section{Performance Analytics}

Powerful behaviour change is possible when data are collected and interpreted to highlight gaps between our intended and actual performance. In healthcare, population-based administrative data are gathered at the point of care for billing purposes and analytics can tell us if we are achieving clinical objectives. Adoption of such data-oriented practices, or performance analytics, in higher education is relatively recent [33]. Historically, most program evaluations have not addressed outcomes beyond the second level of skills based on the Kirkpatrick model $[34,35]$, but performance analytics may hold the key to this.

For example, within LMS, tracking learner behaviours and their engagement with faculty-generated questions or materials could be quite useful for determining student engagement with both the teacher's content and the LMS. Data including number of clicks, time spent on a page, and retention of concepts are correlated with academic performance to provide learning interventions in a timely fashion [36]. Although these data have been used for improving learners' skills and knowledge, faculty development can benefit from the identification of faculty members who need improvement in their online teaching skills. 
Table 1 Overview of future trends in faculty development for health sciences education

\begin{tabular}{|c|c|}
\hline Trend & Potential impact \\
\hline Just-in-time faculty development & $\begin{array}{l}\text { JiT has a focus on "need" in various formats: perceived, on-demand, } \\
\text { or push. Ideally, content is provided in small amounts. Faculty } \\
\text { development curriculum can harness micro-content to utilize small } \\
\text { chunks of learning, (i.e. micro-learning). This can facilitate asyn- } \\
\text { chronous fast-forwarded learning. Online technology identifies } \\
\text { learning needs, drives the process to track faculty members, and } \\
\text { consequently provides credentials for their learning. Certification } \\
\text { on paper or digital can be converted to digital badges to represent } \\
\text { particular competence as a learning outcome. }\end{array}$ \\
\hline
\end{tabular}

Performance analytics

Virtual communities of practice $(\mathrm{CoPs})$

Going from streaming to interactivity

Building capacity for teaching (and presenting) with technology
Each learner may have millions of touch points (i.e. data points) per course. Systems for effective performance analytics are complex and depend upon collecting the right data, analyzing to formulate appropriate insights, and acting in response to these insights so there is an observable positive impact. It is important to ensure that the design of faculty development programs include data analytics capability in order to interpret the relationship between learner behaviours and outcome. In faculty development programs, the use of performance analytics can ensure faculty training is both effective and relevant.

One way to harness the power of CoPs is to cultivate teaching CoPs connecting often geographically disparate faculty members, a phenomenon already occurring in clinical settings. Faculty development programs, too, can conduct educational programming by participating in normal group functioning of CoPs. The rapid pace of current practice means faculty members are facing real challenges in continuing to participate in synchronous activities. How do we best ensure ongoing PFD in this new reality? ALiEM [12], a blog-based online resource, and JGME [13], a hot topic journal club session with open-access articles, are two examples of virtual CoPs [14-16].

Two of the key barriers to participation in faculty development activities are lack of time and logistics [17]. The use of streaming video may help to overcome these barriers by affording opportunities such as delivery of bite-size content, participation at a convenient time, engagement of and dissemination to a targeted or broad audience, as well as reaching a geographically dispersed audience. The addition of interactivity to streaming video can lead to a more robust educational experience through increased engagement, motivation, and retention of learning [18].

As digitally experienced teachers who are more well-versed with these techniques enter the ranks of academic medicine, we are seeing the adoption of many new educational technologies. Although some may discount these advancements by the so-called millennials and digital natives, it is clear that the everyday familiarity we have with these technologies is creeping into medical science education [19]. For those who are unwilling or unable to adapt to this new landscape, there is the possibility of marginalization of great teachers with much to share.

\section{Leveraging Performance Analytics for Faculty Development}

Self-reported behaviour change is the most commonly reported outcome of faculty development [37]. Capacity building at a faculty level combined with the integration of data analytics systems into academic institutions can provide higher education with unprecedented access to information that can be prescriptive (i.e. action-orientated such as selecting which courses to discontinue), predictive (i.e. suggestions for future actions such as which learners may require additional support), or descriptive (i.e. reflections on past actions such as correlating a learner's evaluation outcomes with page views for online content) [38].

An important aspect for faculty capacity building will be around how we collect and meaningfully interpret data. Data can help teachers to better align with learner needs, help identify opportunities for change, or stop ineffective activities. 
Although there are many opportunities in the use of performance analytics to improve higher education [39], we also emphasize that this shift requires additional human resources to manage data systems, educator engagement to identify important measures to be tracked, and faculty development programs to support actions taken in response to data. Working with educators to develop dashboards that quickly help them interpret data can empower faculty members to respond to these insights, and allow them to cycle back to examine the impact of that response [40].

\section{Examples of Performance Analytics for Health Professionals}

Although most faculty development programs have not started to utilize performance analytics yet, there are other examples from various fields which may guide and inform it. The success of a performance analytics approach in higher education is contingent upon the use of a relevant insight generation framework that provides indications of future learner outcomes. For example, the University of South Pacific [41] has developed an "early warning system" to predict student performance based on online engagement whereas others have developed e-rubrics which incorporate data from online learning systems to make it actionable [42]. Perhaps similar to how physicians are starting to use their performance data to improve clinical practice [43, 44], teachers can meet together to examine their own learning analytics to compare practice and improve teaching performance via discussion and peer support.

\section{Virtual Communities of Practice in Society}

CoPs were first described by Lave and Wenger in 1991 [45], and later modernized [46-48]. Conceptually, CoPs are groups that may evolve spontaneously or be cultivated intentionally as a result of the need to solve a unique problem or accomplish a particular task.

In a world connected with instant messaging apps and social media, countless CoPs have been created in all aspects of modern life. As originally defined, these CoPs function well in solving common problems together as virtual entities or as virtual representations of real groups. Groups that exist primarily in virtual platforms are commonly referred to as a "hive", a term that describes a large group of busy individuals working towards a common goal.

\section{Leveraging Virtual Communities for Faculty Development}

Many educators (especially clinician educators) accept social media and instant chat groups as mainstream modes of gaining continuing education [49-51], socialization, and communication. In many medical school programs, both open and closed digital platforms are being used to create a $\mathrm{CoP}$ amongst often geographically disparate faculty members [52]. The design of these platforms is intended to be versatile and intuitive, making them highly accessible as tools in continuing professional and faculty development.

Virtual CoP in faculty development can include WhatsApp [53] group chats [54] and Facebook [55] groups or pages [56], depending on the needs of its members. They can also entail looser (open) associations that, in keeping with CoP principles, allow members to enter and exit, as in \#medtwitter virtual conversations. For more formal programs in faculty development, Slack (i.e. channel-based messaging platform) is increasingly being harnessed as a powerful collaboration solution which augments effective team communication via organized conversations [57]. An example of this is the Academic Life in Emergency Medicine group's Faculty Incubator Program [58].

\section{Examples of Virtual Communities for Health Professionals}

The recent COVID-19 pandemic [59] has resulted in an urgent need for faculty development, because faculty members faced an immediate shift from in-person teaching in medical school UGME and PGME to virtual programming. The ALiEM team [60] and the McMaster University Program for Faculty Development (MacPFD) [61] connected pre-existing virtual $\mathrm{CoP}$ with dozens of educators at the ready. As a consequence, even with apprentices in the mix, they were able to publish extremely timely and scholarly digital outputs aimed at skills that become newly requisite to all teaching faculty (see Table 2). Within four days of social isolation protocols being enacted at institutions across North America, they rapidly created and disseminated this content through @ALiEMteam and @MacPFD twitter accounts which offered how-to tips and guidance for educators who needed to pivot quickly to digital curricula and online teaching solutions in the wake of COVID-19 and the cancellation of in-person classwork. Without the pre-existing CoP behind these intentionally organized groups, this type of JiT faculty development could not have occurred.

\section{Interactivity with Streaming Videos}

Streaming video has gained widespread popularity over the past two decades in large part due to the ease of use and access, high-speed internet connections, mobile devices, downloading or viewing content offline is not required, etc. The ability to stream video enables content creators to share 


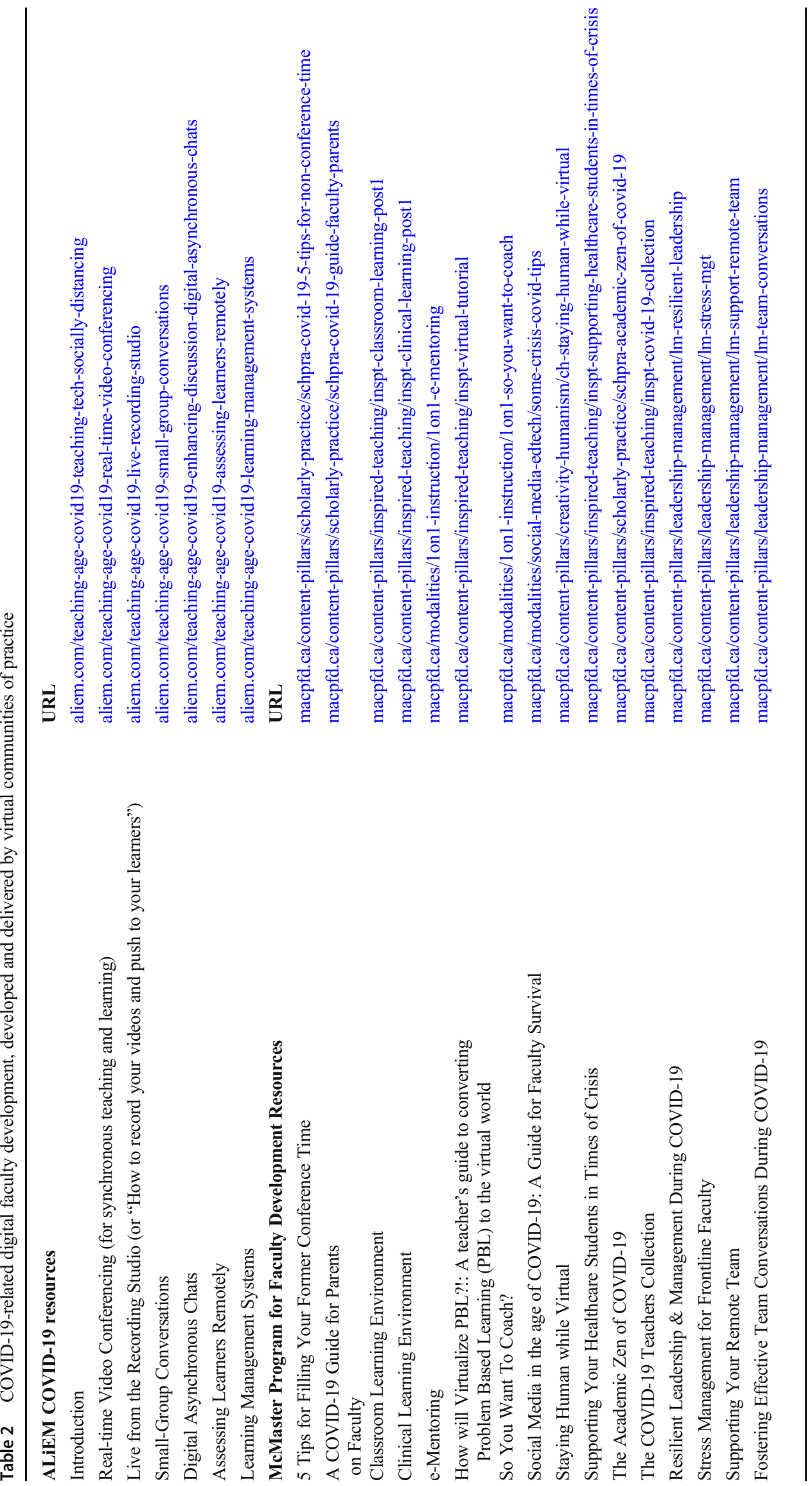


their content with a broad, large audience — and for educators, this means our learners.

The technological capabilities of these platforms have advanced from streaming video to include interactivity ranging from commenting and liking posts to live Q\&A, polling, and chat. The ability to interact with streaming media and others who are viewing the media at the same time has transformed what once was a passive experience into an active experience. Interactive live streaming is an emerging trend that creates an immersive experience where the viewer participates in the livestream (and may even be involved in the co-creation of it), creates connections, and can build community with other viewers.

\section{Interactivity in Faculty Development}

The ability to stream video and simultaneously engage audiences by employing a variety of tools such as live polling and chat makes interactive streaming video an attractive educational tool. Until the COVID-19 pandemic, many faculties of medicine or health professions seemingly shied away from the use of these technologies - with hospitals or clinics even preventing their use due to firewalls or other digital security measures. However, since the beginning of the COVID-19 pandemic, there has been a marked shift in technology adoption for healthcare purposes - which bodes well for delivery of online faculty development initiatives and engagement by faculty who are in clinical settings and/or are geographically dispersed.

The use of tools such as YouTube [15], Vimeo [62], and Zoom [63] is more widely adopted in healthcare contexts and consequently more accessible to us within health professions faculty development. Live streaming video is being used for a variety of purposes in the higher education landscape ranging from delivery of live-streamed lectures in undergraduate medical education [64], broadcasting of surgical performance for surgical tele-mentoring [65] to continuing professional development. Furthermore, the use of streaming media is frequently being used by healthcare professionals to learn both technical and non-technical skills $[66,67]$. While these technologies are already in use at institutions, many faculty members do not use them on a regular basis and as a result may lack comfort and confidence in using these tools for educational purposes. Furthermore, there are opportunities for continued development of faculty in using these technologies as well as using these platforms for delivery of faculty development.

\section{Examples of Interactivity for Health Professionals}

While not groundbreaking, these technologies allow for broadcast in an accessible and cost-effective way that lends themselves to be quite useful for faculty developers in the health professions. One of the benefits of platforms like
Facebook [55] and YouTube [15] is the ability to livestream to large audiences. A recent example of this was the creation of PathCast which broadcasts pathology lectures to an international audience that can interact via the platforms' chat feature [68].

The COVID-19 pandemic [69] triggered a rapid shift from in-person meetings and events to online platforms. For example, the McMaster Faculty of Health Sciences Research, Innovation and Theory (MERIT) Program recently delivered a monthly round on "Data, Data, Data" via a livestream using Zoom [70]. Another recent example at McMaster University was a Town Hall meeting for faculty and students that coupled live stream video with interactive presentation tools to provide an avenue for interactivity and co-creation of the session by prioritizing the questions the audience wanted addressed during the livestream.

\section{Investing in Champions Who Teach (and Present) via Technology}

Almost a decade ago, Charles Prober (prominent educator at Stanford) and Salman Khan (of the Khan Academy, a nonprofit organization focused on creating online education that allows teachers to engage in flipped classrooms in K-12) wrote about the promise of digital online recordings and how this could revolutionize the world of medical education [71]. At the time, they wrote: "The authors argue that the modern digitally empowered learner, the unremitting expansion of biomedical knowledge, and the increasing specialization within the practice of medicine drive the need to reimagine medical education" [71]. They proposed that by archiving the best teachers from around the world, we could increase efficiency within our medical training programs and provide learners higher-order learning experiences. Rather than watch a lecture, students might pre-learn the material via YouTube videos, and then engage in higher-order thinking and discussions around cases via problem-based learning $[72,73]$ or other types of case-based learning [74]. Leveraging existing online resources can supplement flipped classroom strategies [75-78].

\section{Champions of Teaching via Technology in Faculty Development}

Current champions who integrate internet-based technology into their teaching turn to YouTube [15], webinars, Massive Open Online Courses (MOOCs), and podcasts. Indeed, easily accessible training videos are now a daily phenomenon for most individuals in our society on any topic. Most people likely assume one can simply go to their computer to learn to do almost any simple task (e.g. how to record video lecture using a smartphone). Indeed, for most everyday tasks, the 
expertise of the instructor is soon realized by the efficiency of learning and skill acquisition of the viewer. In professional contexts, expertise may need to be independently validated, yet medical science education has not yet realized the potential of these technologies.

\section{Examples of Teaching via Technology for Health Professionals}

A blend of many of the above strategies (just-in-time, fostering a virtual $\mathrm{CoP}$, facilitating interactivity) are best exemplified by online chats. For instance, \#HMIChat is a monthly tweet chat focused on health professions education, curated by Harvard Macy Institute community members and open to the global Twitter community [79]. Another example \#MedEdChat is a Twitter-based weekly online discussion forum $[79,80]$ whose topics are dynamic and informed by its community, with chat transcripts also posted online for asynchronous benefit (see, bit.ly/MedEdTwitter). Since the technological barrier for participating in Twitter is relatively low, individuals of a wide range of technological comfort will often tune in (either reading or "lurking"). Often individuals who regularly follow along in these chats will eventually participate and may even guest moderate.

\section{Discussion}

The future of faculty development for medical science educators is bright. New technologies can connect educators better with both students and colleagues. The greatest challenge that we face is effectively encouraging the adoption of these new technologies for continuous professional development (including, but not limited to, faculty development).

Fig. 1 Future trends and possibilities in faculty development

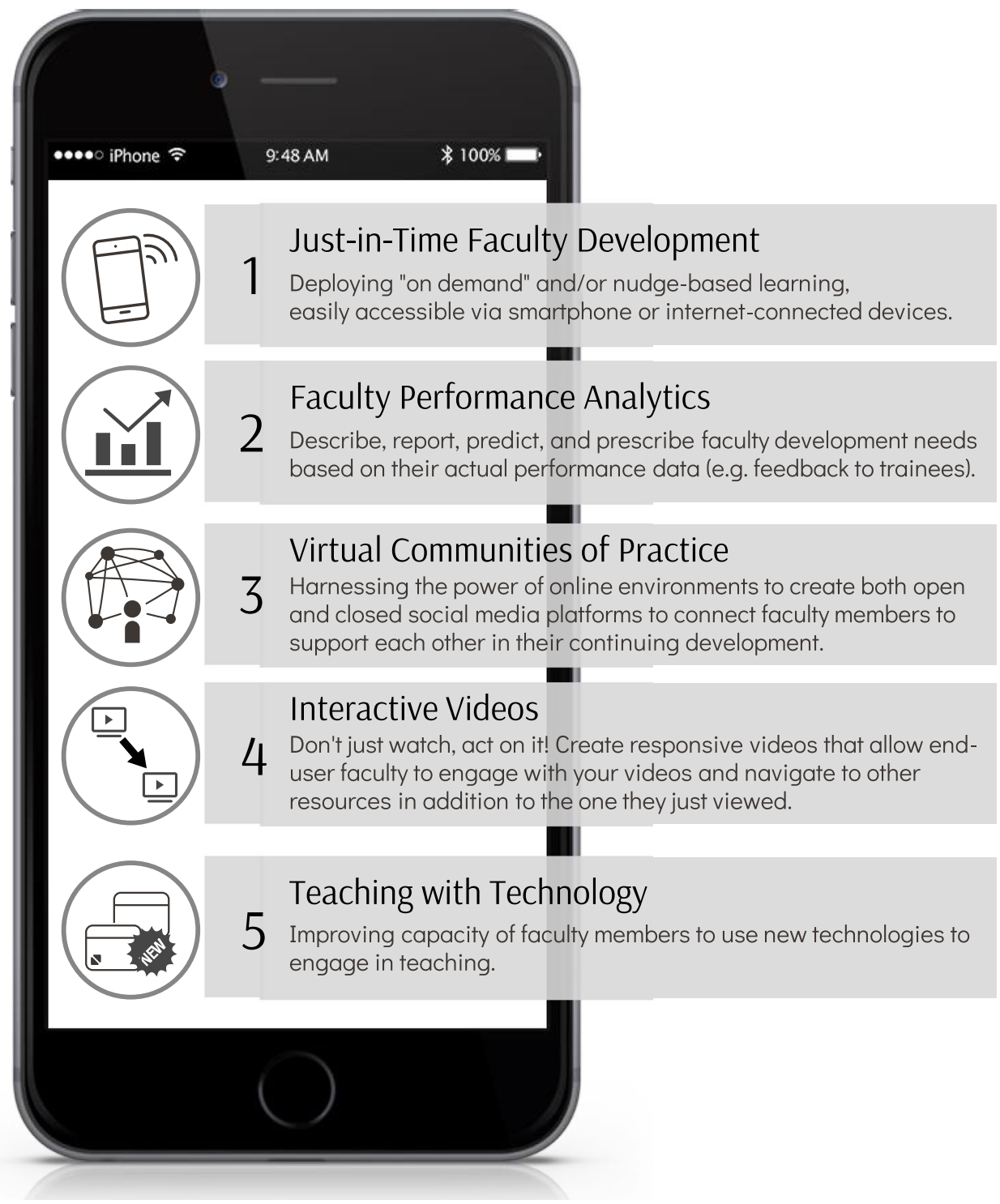


We have detailed some near future trends that faculty development programs should consider when designing their programming. Figure 1 summarizes these trends and possibilities. In addition to these methods, we should still attend to more standard technologies like video streaming (e.g. YouTube, Vimeo), podcasting, website construction (including blogging), and possibly app development.

Not only must we align our faculty development to both current and future trends, but we must also ensure that we ourselves (as faculty developers) harness the power of these technologies to create our faculty development projects. It should not be only our students and trainees who benefit from these new technologies; harnessing these technologies may be the secret to addressing problems that commonly occur in faculty development (such as disengagement and inability to incorporate new learning objectives into faculty lives). The same technological trends that improve learning outcomes for trainees are likely many of the same trends that can similarly apply to faculty development.

The pressures to create technologically savvy content for our students are very similar to those that exist to create better digitally enhanced faculty development. Trying to increase engagement via gamifying interactive video content is a good example of what the future expects for faculty development. Strategically accessing just-in-time content via smartphone, as an example, may apply equally to the busy college senior and the mid-career faculty member who may have multiple work and personal commitments. Content for either person is likely going to have to fit within their increasingly busy lives.

We expect these trends will be present in many faculty development programs. Organizational frameworks such as Technological Pedagogical Content Knowledge (TPACK) [81] might be used to help reveal gaps in current programming and encourage faculty developers to consider incorporating different modalities and approaches into their programs. It is also important to expand the scope of what we consider in the auspices of faculty development, which in the medical sciences has gravitated towards teacher training. Modern thought leaders in this space have been increasingly advocating a pivot in faculty development from isolated workshops on pedagogical skills, to methods that support more diverse skills encompassing multiple aspects of personal development, in a longitudinal design $[82,83]$. In our modern time, this will require the ongoing support of our medical sciences faculty members' knowledge, skills, and attitudes on the use of new educational and healthcare technologies [84].

We have described upcoming trends to faculty development. While each trend can "stand-alone" to support faculty development, their interconnection can build an even greater infrastructure which could be termed a faculty development ecosystem. This ecosystem can host micro-learning designed interactive video and educational resources to support the JiT approach for faculty members. Additionally, the ecosystem can serve as an online $\mathrm{CoP}$ which is open to everyone to build capacity for teaching.

\section{Conclusion}

Faculty development must evolve and change to meet the needs of the present time. Future faculty members who are more familiar with technology will expect faculty development methods aligned with their daily habits of technology use. The trends described in this paper have high potential to fulfill those needs of future faculty members, and to inform the future of faculty development.

Acknowledgements We would like to thank the rest of the Program for Faculty Development team for their hard work. Without them, it is unlikely that we would be able to pull off the great programming we have planned.

Code Availability Not applicable

Authors' Contributions All of the authors have contributed to the design and draft of the paper, written a section, read the final version, and confirmed the content of this study.

Data Availability Not applicable

\section{Compliance with Ethical Standards}

Conflict of Interest Dr. Yilmaz receives the TUBITAK Postdoctoral Fellowship grant. Dr. Chan receives a stipend for her duties as the assistant dean for the Program for Faculty Development at McMaster University. She also has received a PSI foundation grant for her work in social media and knowledge translation. Dr. Tong receives a stipend for her role as the Faculty Development Coordinator at the Waterloo Regional Campus, McMaster University. The remaining authors do not have competing interests.

Ethics Approval N/A

\section{References}

1. Moore AH, Moore JF, Fowler SB. Faculty development for the net generation [Internet]. Educ. Net Gener. 2005 [cited 2020 Jan 22]. Available from: https://www.educause.edu/research-andpublications/books/educating-net-generation/faculty-developmentnet-generation.

2. Triola MM, Huwendiek S, Levinson AJ, Cook DA. New directions in e-learning research in health professions education: report of two symposia. Med Teach. 2012;34:e15-20.

3. de Giovanni D, Roberts T, Norman G. Relative effectiveness of high- versus low-fidelity simulation in learning heart sounds. Med Educ. 2009;43:661-8.

4. Gillan C, Papadakos J, Brual J, Harnett N, Hogan A, Milne E, et al. Impact of high-fidelity e-learning on knowledge acquisition and satisfaction in radiation oncology trainees. Curr Oncol. 2018;25: e533-8. 
5. Levinson AJ, Weaver B, Garside S, McGinn H, Norman GR. Virtual reality and brain anatomy: a randomised trial of e-learning instructional designs. Med Educ. 2007;41:495-501.

6. Matsumoto ED, Hamstra SJ, Radomski SB, Cusimano MD. The effect of bench model fidelity on endourological skills: a randomized controlled study. J Urol. 2002;167:1243-7.

7. Border S. Assessing the role of screencasting and video use in anatomy education. Adv Exp Med Biol. 2019;1171:1-13.

8. Finn GM, Matthan J. Pedagogical perspectives on the use of technology within medical curricula: moving away from norm driven implementation. Adv Exp Med Biol. 2019;1120:55-65.

9. Pickering JD, Swinnerton BJ. Exploring the dimensions of medical student engagement with technology-enhanced learning resources and assessing the impact on assessment outcomes. Anat Sci Educ. 2019;12:117-28.

10. Rogers EM. Diffusion of innovations (5th ed.). New York: Free Press; 2003.

11. Davis FD, Bagozzi RP, Warshaw PR. User acceptance of computer technology: a comparison of two theoretical models. Manag Sci. 1989;35:982-1003.

12. Steinert Y, Mann K, Centeno A, Dolmans D, Spencer J, Gelula M, et al. A systematic review of faculty development initiatives designed to improve teaching effectiveness in medical education: BEME guide no. 8. Med Teach. 2006;28:497-526.

13. Steinert Y, Mann K, Anderson B, Barnett BM, Centeno A, Naismith L, et al. A systematic review of faculty development initiatives designed to enhance teaching effectiveness: a 10-year update: BEME guide no. 40. Med Teach. 2016;38:769-86.

14. Google. Google - about Google, our culture \& company news [Internet]. [cited 2020 Mar 31]. Available from: https://about. google. Accessed 31 Mar 2020.

15. Youtube. About YouTube - YouTube [Internet]. [cited $2020 \mathrm{Mar}$ 31]. Available from: https://www.youtube.com/about/. Accessed 31 Mar 2020.

16. Twitter. About Twitter [Internet]. [cited 2020 Mar 31]. Available from: https://about.twitter.com/en us.html. Accessed 31 Mar 2020.

17. Kim S, Noveck H, Galt J, Hogshire L, Willett L, O'Rourke K. Searching for answers to clinical questions using google versus evidence-based summary resources: a randomized controlled crossover study. Acad Med J Assoc Am Med Coll. 2014;89:940-3.

18. Eva KW, Regehr G. "I'll never play professional football" and other fallacies of self-assessment. J Contin Educ Health Prof. 2008;28: 14-9.

19. Patocka C, Lin M, Voros J, Chan T. Point-of-care resource use in the emergency department: a developmental model. AEM Educ Train. 2018;2:221-8.

20. Eva KW, Regehr G. Knowing when to look it up: a new conception of self-assessment ability. Acad Med J Assoc Am Med Coll. 2007;82:S81-4.

21. Emerson LC, Berge ZL. Microlearning: Knowledge management applications and competency-based training in the workplace. 2018 [cited 2020 Jan 24]; Available from: http://mdsoar.org/handle/ $11603 / 16035$.

22. Fornari A, Corral J. Just in time teaching (JITT) tips. Royal College of Physicians and Surgeons of Canada; 2019. Available from: http://www.royalcollege.ca/rcsite/events/icre/facdev-conference-e. Accessed 26 Mar 2020.

23. Novak GM. Just-in-time teaching. New Dir. Teach. Learn. 2011;128:63-73.

24. Varney J. The trend for just-in-time learning. Hum Resour Mag. 2018;23:4-6.

25. Knutson A, Park ND, Smith D, Tracy K, Reed DJW, Olsen SL. Just-in-time training: a novel approach to quality improvement education. Neonatal Netw. 2015;34:6-9.

26. Hudspeth D. Just-in-time education. Educ Technol. 1992;32:7-11.
27. Papanagnou D, Stone D, Chandra S, Watts P, Chang AM, Hollander JE. Integrating telehealth emergency department follow-up visits into residency training. Cureus. 2018;10:e2433.

28. Boileau E, St-Onge C, Audetat M-C. Utility of a mobile application using an algorithmic approach to clinical reasoning difficulties. Can Fam Physician. 2017;63:S36.

29. Cook DA. Faculty development online. In: Steinert Y, editor. Fac Dev Health Prof [Internet]. Dordrecht: Springer; 2014. p. 217-41. Available from:. https://doi.org/10.1007/978-94-007-7612-8 11.

30. Karademos J, Rodriguez C, Siddiqui M, Naples R, Papanagnou D. Just in time: a faculty development primer to help prepare core faculty for clinical teaching shifts. West J Emerg Med. 2019;20: S27.

31. Nelson DM, Joswiak ME, Brake KA. "Just in time" training for novice preceptors. J Nurses Prof Dev. 2019;35:228-31.

32. Corral J, Post MD, Bradford A. Just-in-time faculty development for pathology small groups. Med Sci Educ. 2018;28:11-2.

33. Attaran M, Stark J, Stotler D. Opportunities and challenges for big data analytics in US higher education: a conceptual model for implementation. Ind High Educ. 2018;32:169-82.

34. Yardley S, Teunissen PW, Dornan T. Experiential learning: transforming theory into practice. Med Teach. 2012;34:161-4.

35. Yardley S, Dornan T. Kirkpatrick's levels and education 'evidence': Kirkpatrick’s levels in education. Med Educ. 2012;46:97106.

36. Romero C, Ventura S, García E. Data mining in course management systems: Moodle case study and tutorial. Comput Educ. 2008;51:368-84.

37. Leslie K, Baker L, Egan-Lee E, Esdaile M, Reeves S. Advancing faculty development in medical education: a systematic review. Acad Med. 2013;88:1038-45.

38. Daniel B. Big Data and analytics in higher education: opportunities and challenges: the value of Big Data in higher education. Br J Educ Technol. 2015;46:904-20.

39. Zilvinskis J, Willis J, Borden VMH. An overview of learning analytics: an overview of learning analytics. New Dir High Educ. 2017;2017:9-17.

40. Lucio R, Campbell M, Detres M, Johnson H. Using dashboards to engage faculty in improving academic programs and courses. Valencia, Spain; 2018 [cited 2020 Mar 26]. p. 1844-52. Available from: http://library.iated.org/view/LUCIO2018USI.

41. Jokhan A, Sharma B, Singh S. Early warning system as a predictor for student performance in higher education blended courses. Stud High Educ. 2019;44:1900-11.

42. Dimopoulos I, Petropoulou O, Retalis S. Assessing students' performance using the learning analytics enriched rubrics. Proc Third Int Conf Learn Anal Knowl - LAK 13 [Internet]. Leuven, Belgium: ACM Press; 2013 [cited 2020 Mar 26]. p. 195. Available from: http://dl.acm.org/citation.cfm?doid=2460296.2460335.

43. Cooke LJ, Duncan D, Rivera L, Dowling SK, Symonds C, Armson $\mathrm{H}$. How do physicians behave when they participate in audit and feedback activities in a group with their peers? Implement Sci. 2018;13:104.

44. Cooke LJ, Duncan D, Rivera L, Dowling SK, Symonds C, Armson H. The Calgary Audit and Feedback Framework: a practical, evidence-informed approach for the design and implementation of socially constructed learning interventions using audit and group feedback. Implement Sci. 2018;13:136.

45. Lave J, Wenger E. Situated learning: legitimate peripheral participation. Cambridge, UK: Cambridge University Press; 1991.

46. Bourhis A, Dubé L, Jacob R. The success of virtual communities of practice : the leadership factor [Internet]. 2005 [cited 2020 Mar 26]. Available from: https://www.semanticscholar.org/paper/TheSuccess-of-Virtual-Communities-of-Practice-\%3A-Bourhis-Dub\% C3\%A9/3d2462cc548a01c644ec26d678de3250ef9a8da0. 
47. Dube L, Bourhis A, Jacob R. Towards a typology of virtual communities of practice. Interdiscip J Inf Knowl Manag. 2006;1:06993.

48. Dubé L, Bourhis A, Jacob R. The impact of structuring characteristics on the launching of virtual communities of practice. J Organ Change Manag. 2005;18:145-66.

49. Bolderston A, Watson J, Woznitza N, Westerink A, Di Prospero L, Currie G, et al. Twitter journal clubs and continuing professional development: an analysis of a \#MedRadJClub tweet chat. Radiogr Lond Engl 1995. 2018;24:3-8.

50. Melvin L, Chan T. Using Twitter in clinical education and practice. J Grad Med Educ. 2014;6:581-2.

51. Thoma B, Joshi N, Trueger NS, Chan TM, Lin M. Five strategies to effectively use online resources in emergency medicine. Ann Emerg Med. 2014;64:392-5.

52. Yarris LM, Chan TM, Gottlieb M, Juve AM. Finding your people in the digital age: virtual communities of practice to promote education scholarship. J Grad Med Educ. 2019;11:1-5.

53. About WhatsApp [Internet]. WhatsApp.com. [cited 2020 Mar 31]. Available from: https://www.whatsapp.com/about/.

54. Cansoy R. Teachers' professional development: the case of WhatsApp. J Educ Learn. 2017;6:285.

55. Facebook. About Facebook [Internet]. Facebook. [cited 2020 Mar 31]. Available from: https://about.fb.com/.

56. Staudt D, Clair NS, Martinez EE. Using Facebook to support novice teachers. New Educ. Routledge. 2013;9:152-63.

57. Slack. What is Slack? [Internet]. Slack Help Cent. [cited 2020 Mar 31]. Available from: https://slack.com/intl/en-ca/help/articles/ 115004071768-What-is-Slack-.

58. Chan TM, Gottlieb M, Sherbino J, Cooney R, Boysen-Osborn M, Swaminathan A, et al. The ALiEM Faculty Incubator: a novel online approach to faculty development in education scholarship. Acad Med. 2018;93:1497-502.

59. Wu Z, McGoogan JM. Characteristics of and important lessons from the coronavirus disease 2019 (COVID-19) outbreak in China: summary of a report of 72314 cases from the Chinese Center for Disease Control and Prevention. JAMA [Internet]. 2020 [cited 2020 Mar 26]; Available from: https://jamanetwork. com/journals/jama/fullarticle/2762130.

60. Lin M. About ALiEM [Internet]. ALiEM. [cited 2020 Mar 31]. Available from: https://www.aliem.com/about-us/.

61. MacPFD. MacPFD Team Site [Internet]. [cited 2020 Mar 31]. Available from: https://www.macpfd.ca/home.

62. Vimeo. About Vimeo [Internet]. [cited 2020 Mar 31]. Available from: https://vimeo.com/about.

63. Zoom. Video conferencing, web conferencing, webinars, screen sharing [Internet]. Zoom Video. [cited 2020 Mar 31]. Available from: https://zoom.us/.

64. Sandhu A, Fliker A, Leitao D, Jones J, Gooi A. Adding livestreaming to recorded lectures in a non-distributed pre-clerkship medical education model. Stud Health Technol Inform. 2017;234: 292-7.

65. Lin C-C, Chen Y-P, Chiang C-C, Chang M-C, Lee OK-S. Broadcast of surgery performance and intra-operative imaging data: a research of real-time streaming system augmented in the hybrid operating room (Preprint). JMIR Med Inform [Internet]. 2020 [cited 2020 Mar 29]; Available from: http://preprints.jmir.org/ preprint/18094/accepted.
66. Tackett S, Slinn K, Marshall T, Gaglani S, Waldman V, Desai R. Medical education videos for the world: an analysis of viewing patterns for a YouTube channel. Acad Med. 2018;93:1150-6.

67. Topps D, Helmer J, Ellaway R. YouTube as a platform for publishing clinical skills training videos. Acad Med. 2013;88:192-7.

68. Madrigal E, Mannan R. pathCast: an interactive medical education curriculum that leverages live streaming on Facebook and YouTube. Acad Med. 2020;95(5):744-50.

69. WHO. Coronavirus disease (COVID-19) pandemic [Internet]. [cited 2020 Mar 31]. Available from: https://www.who.int/ emergencies/diseases/novel-coronavirus-2019.

70. MERIT. We're taking @ MERIT McMaster Rounds fully virtual for the next little while [Internet]. Were Tak. MERITMcMaster Rounds Fully Virtual Little While. [cited 2020 Apr 1]. Available from: https://twitter.com/MERIT McMaster/status/ 1239984045301342214.

71. Prober CG, Khan S. Medical education reimagined: a call to action Acad Med. 2013;88:1407-10.

72. Barrows HS. Practice-based learning: Problem-based learning applied to medical education. Springfield, IL: Southern Illinois University, School of Medicine; 1994.

73. Barrows HS, Tamblyn RM. Problem-based learning: an approach to medical education (Vol 1). New York: Springer Publishing Company; 1980.

74. Thistlethwaite JE, Davies D, Ekeocha S, Kidd JM, MacDougall C, Matthews $\mathrm{P}$, et al. The effectiveness of case-based learning in health professional education. A BEME systematic review: BEME guide no. 23. Med Teach. 2012;34:e421-44.

75. Akçayır G, Akçayır M. The flipped classroom: a review of its advantages and challenges. Comput Educ. 2018;126:334-45.

76. Betihavas V, Bridgman H, Kornhaber R, Cross M. The evidence for "flipping out": a systematic review of the flipped classroom in nursing education. Nurse Educ Today. 2016;38:15-21.

77. Chen F, Lui AM, Martinelli SM. A systematic review of the effectiveness of flipped classrooms in medical education. Med Educ. 2017:51:585-97.

78. Hew KF, Lo CK. Flipped classroom improves student learning in health professions education: a meta-analysis. BMC Med Educ. 2018; $18: 38$.

79. Chan TM, Stukus D, Leppink J, Duque L, Bigham BL, Mehta N, et al. Social media and the 21st-century scholar: how you can harness social media to amplify your career. J Am Coll Radiol JACR. 2018;15:142-8.

80. Djuricich AM, Zee-Cheng JE. Live tweeting in medicine: "Tweeting the meeting". Int Rev Psychiatry Abingdon Engl. 2015;27:133-9.

81. Mishra P, Koehler MJ. Technological Pedagogical Content Knowledge: a framework for teacher knowledge. Teach Coll Rec. 2006;108:1017-54.

82. Steinert Y. Faculty development: from rubies to oak. Med Teach 2020;42:429-35.

83. Steinert $Y$. Perspectives on faculty development: aiming for $6 / 6$ by 2020. Perspect Med Educ. 2012;1:31-42.

84. Shulman LS. Those who understand: knowledge growth in teaching. Educ Res. 1986;15:4-14.

Publisher's Note Springer Nature remains neutral with regard to jurisdictional claims in published maps and institutional affiliations. 